\title{
A SMALL REMARK ON THE FILTERED $\varphi$-MODULE OF FERMAT VARIETIES AND STICKELBERGER'S THEOREM
}

\author{
By
}

Go YAMASHITA ${ }^{\dagger}$

\begin{abstract}
We show that the weakly admissibility of the filtered $\varphi$-module with coefficients of Fermat varieties in the sense of Fontaine essentially expresses Stickelberger's theorem in Iwasawa theory. In particular, it gives us a simple re-proof of the weakly admissibility of it.
\end{abstract}

This short paper is essentially a letter to Noriyuki Otsubo in July/2013.

\section{Introduction}

Let $V=V_{d}^{n}$ be the relatively $n$-dimensional Fermat variety of degree $d$ over $\mathbf{Z}\left[\mu_{d}\right]$, i.e., the variety defined by the equation

$$
X_{0}^{d}+X_{1}^{d}+\cdots+X_{n+1}^{d}=0
$$

in $\mathbf{P}_{\mathbf{Z}\left[\mu_{d}\right]}^{n+1}$. For a prime number $p$ which does not divide $d$, we fix an embedding of $\mu_{d}$ into an algebraic closure $\overline{\mathbf{Q}_{p}}$ of $\mathbf{Q}_{p}$. Let $\mathbf{F}_{q}$ denote the residue field of $K:=\mathbf{Q}_{p}\left(\mu_{d}\right)$ and $q=p^{f}$. Note that $d$ divides $q-1$. The fractional field of the ring of Witt vectors $W:=W\left(\mathbf{F}_{q}\right)$ with coefficients in $\mathbf{F}_{q}$ is canonically isomorphic to $K$ since $p$ does not divide $d$. Let $\sigma$ denote the Frobenius of $K$.

We consider the following two cohomology groups: Firstly, the de Rham cohomology group $H_{\mathrm{dR}}:=H_{\mathrm{dR}}^{n}\left(V_{K} / K\right)$ of $V_{K}:=V \otimes_{\mathbf{Z}\left[\mu_{d}\right]} K$, which is a finite dimensional $K$-vector space equipped with the Hodge filtration $\left\{\mathrm{Fil}^{i} H_{\mathrm{dR}}\right\}_{i}$. Secondly, the crystalline cohomology group $H_{\text {crys }}:=H_{\text {crys }}^{n}\left(V_{0} / W\right) \otimes_{W} K$ of

2010 Mathematics Subject Classification: Primary 11D41, Secondary 11R23, 14F30.

Key words and phrases: Fermat varieties, Stickelberger's theorem, filtered $\varphi$-modules, Newton polygon, Hodge polygon, crystalline cohomology, Jacobi sum, Gauss sum.

$\dagger$ Supported by TOYOTA Central R\&D Labs., Inc., and partially supported by JSPS Grant-in-Aid for Scientific Research (C), 15K04781.

Received January 15, 2016.

Revised May 13, 2016. 
$V_{0}:=V \otimes_{\mathbf{Z}\left[\mu_{d}\right]} \mathbf{F}_{q}$, which is a finite dimensional $K$-vector space equipped with the $\sigma$-semi-linear crystalline Frobenius action $\varphi$. Via Berthelot-Ogus isomorphism [BO], the pair $\left(H_{\mathrm{dR}}, H_{\text {crys }}\right)$ is a filtered $\varphi$-module in the sense of Fontaine $([\mathrm{F}])$. Note that the cohomology groups of other degrees $H_{\bullet}^{q}(q \neq n, \bullet=\mathrm{dR}$, crys $)$ are just zero $(q$ : odd) or Tate objects $(q$ : even) by the weak Lefschetz theorem and the Poincaré duality, hence the structure is well-known.

Both of $H_{\mathrm{dR}}, H_{\text {crys }}$ have natural actions of

$$
S:=\left(\bigoplus_{i=0}^{n+1} \mu_{d}\right) / \Delta\left(\mu_{d}\right),
$$

which are induced by the action on $V$ given by $\left[X_{0}: \cdots: X_{n+1}\right] \mapsto\left[\zeta_{0} X_{0}: \cdots\right.$ : $\left.\zeta_{n+1} X_{n+1}\right]$ for $\left(\zeta_{0}, \ldots, \zeta_{n+1}\right) \bmod \Delta\left(\mu_{d}\right) \in S$, where $\Delta$ is the diagonal homomorphism. This action of $S$ induces an action of $S$ on $V_{0}$ as well. However, the crystalline Frobenius (i.e., induced by the $p$-power map) is not compatible with this action of $S$. Hence, we consider cohomology groups $H_{\mathrm{dR}} \otimes_{\mathbf{Q}_{p}} K$ and $H_{\text {crys }} \otimes_{\mathbf{Q}_{p}} K$ over $K \otimes_{\mathbf{Q}_{p}} K$, where we consider the Hodge filtration and the crystalline Frobenius via the left $K$ of $K \otimes_{\mathbf{Q}_{p}} K$, and the action of $S$ via the right $K$ of $K \otimes_{\mathbf{Q}_{p}} K$. To lighten the notations, we put $\otimes:=\otimes_{\mathbf{Q}_{p}}$ in the following. Then, these cohomology groups $H_{\bullet} \otimes K(\bullet=\mathrm{dR}$, crys $)$ are decomposed as filtered $\varphi$-modules with coefficient $K$ by this action:

$$
H_{\bullet} \otimes K=\bigoplus_{\underline{a} \in X(S)}\left(H_{\bullet} \otimes K\right)_{\underline{a}},
$$

where $X(S)$ denotes the group of the characters of $S$, and $\left(H_{\bullet} \otimes K\right)_{a}$ is the $\underline{a}$-part of $H_{\bullet} \otimes K$, i.e., the sub- $K \otimes K$-module of $H_{\bullet} \otimes K$ on which $\underline{\zeta} \in S$ acts by $\underline{a}(\underline{\zeta})$. Note that $X(S)=\left\{\underline{a}=\left(a_{0}, \ldots, a_{n+1}\right) \in \bigoplus_{i=0}^{n+1} \mathbf{Z} / d \mathbf{Z} \mid \sum_{i=0}^{n+1} a_{i}=0\right\}$ and $\underline{a}(\underline{\zeta})=$ $\underline{\zeta}^{\underline{a}}:=\prod_{i=0}^{n+1} \zeta_{i}^{a_{i}}$. It is well-known that $\left(H_{\bullet} \otimes K\right)_{\underline{a}}$ is free $K \otimes K$-module of rank $\operatorname{rank}_{K \otimes K}\left(H_{\bullet} \otimes K\right)_{\underline{a}}= \begin{cases}1 & a_{0}, \ldots, a_{n+1} \neq 0, \text { or }\left(a_{0}=\cdots=a_{n+1}=0 \text { and } n: \text { even }\right), \\ 0 & \text { otherwise. }\end{cases}$ (See [D, Proposition 7.4] and its erratum). (Note that the total chern class $c\left(V_{\mathbf{Q}}\right)=c_{0}\left(V_{\mathbf{Q}}\right)+\cdots+c_{n}\left(V_{\mathbf{Q}}\right)$ of the tangent bundle of $V_{\mathbf{Q}}:=V \otimes_{\mathbf{Z}} \mathbf{Q}$ is given by $c\left(V_{\mathbf{Q}}\right)=(1+x)^{n+2}(1+d x)^{-1}=\left(1+(n+2) x+\cdots+\left(\begin{array}{c}n+2 \\ n\end{array}\right) x^{n}\right)(1-d a+\cdots+$ $\left.(-1)^{n} d^{n} x^{n}\right)$ with $x:=c_{1}(\mathcal{O}(1))$, hence the Euler characteristic $c_{n}\left(V_{\mathbf{Q}}\right)$ is equal to $c_{n}\left(V_{\mathbf{Q}}\right)=\left(\begin{array}{c}n+2 \\ n\end{array}\right) x^{n}-\left(\begin{array}{c}n+2 \\ n-1\end{array}\right) d x^{n}+\cdots+(-1)^{n} d^{n} x^{n}=x^{n} \frac{(1-d)^{n+2}-1+(n+2) d}{d^{2}}=$ $\frac{1}{d}\left((1-d)^{n+2}-1\right)+n+2$. Thus, the dimension of the primitive part of the cohomology group is $h_{\text {prim }}^{n}=(-1)^{n}\left(c_{n}\left(V_{\mathbf{Q}}\right)-(n+1)\right)=\frac{1}{d}\left((d-1)^{n+2}-(-1)^{n}\right)+$ $(-1)^{n}$. On the other hand, the number of $\underline{a} \in X(S)$ with $a_{0}, \ldots, a_{n+1} \neq 0$ 
is $\quad(d-1)^{n+1}-(d-1)^{n}+\cdots+(-1)^{n}(d-1)=\frac{1}{d}\left((d-1)^{n+2}-(-1)^{n}\right)+(-1)^{n}$, which coincides with $h_{\text {prim }}^{n}$, as expected.) In the case where $a_{0}=\cdots=a_{n+1}=0$ and $n$ is even, $\left(H_{\bullet} \otimes K\right)_{a}$ is also a Tate object (See the proof of $[\mathrm{D}$, Proposition 7.4]), hence the structure is well-known. Thus, the remaining part is the case where $a_{0}, \ldots, a_{n+1} \neq 0$.

In the rest of the paper, we assume that $a_{0}, \ldots, a_{n+1} \neq 0$. The pair

$$
\left(\left(H_{\mathrm{dR}} \otimes K\right)_{\underline{a}},\left(H_{\mathrm{crys}} \otimes K\right)_{\underline{a}}\right)
$$

is a filtered $\varphi$-module with coefficient $K$ with $\operatorname{rank}_{K \otimes K}=1$. By using the main theorem of the $p$-adic Hodge theory (See $[\mathrm{T}]$ ), we have:

THEOREM 1.1 (a consequence of $[\mathrm{T}])$. The filtered $\varphi$-module $\left(\left(H_{\mathrm{dR}} \otimes K\right)_{a}\right.$, $\left.\left(H_{\text {crys }} \otimes K\right)_{a}\right)$ with coefficient in $K$ is weakly admissible in the sense of Fontaine $([\mathrm{F}])$.

(Strictly speaking, now we are considering the with-coefficient-version of the weakly admissibility.) In this short paper, we show the weakly admissibility of it without using the difficult theorem of $[\mathrm{T}]$. Our (re-)proof shows that the weakly admissibility of it expresses essentially Stickelberger's theorem in Iwasawa theory (Note that Stickelberger's theorem is based on elementary calculations, and not difficult), and that the concrete content of a special case (i.e., Fermat variety case) of such a general difficult geometric theorem (i.e., the main theorem of the $p$-adic Hodge theory $[\mathrm{T}])$ is of highly arithmetic nature.

\section{2. de Rham Side (Hodge Polygon)}

First, we introduce some notations. For $\underline{a}=\left(a_{0}, \ldots, a_{n+1}\right) \in X(S)$, let $\left\langle a_{i}\right\rangle \in \mathbf{N}$ denote the representative of $a_{i}$ with $1 \leq\left\langle a_{i}\right\rangle \leq d$, and $\langle\underline{a}\rangle:=\frac{1}{d} \sum_{i=0}^{n+1}\left\langle a_{i}\right\rangle \in \mathbf{N}$. For $\alpha \in \mathbf{Q}$, let $0 \leq\{\alpha\}<1$ denote the fractional part of $\alpha$. For $n=n_{0}+n_{1} p$ $+\cdots+n_{f-1} p^{f-1}$ with $0 \leq n_{0}, \ldots, n_{f-1} \leq p-1$, we set $s(n):=n_{0}+\cdots+n_{f-1}$.

Note that $\left(H_{\mathrm{dR}} \otimes K\right)_{a}$ has rank one over $K \otimes K \cong K \times K^{\sigma} \times \cdots \times K^{\sigma^{f-1}}$, and $\left.H_{\mathrm{dR}} \otimes K \cong H_{\mathrm{dR}}^{n}\left(V_{K} / K\right) \oplus H_{\mathrm{dR}}^{n}\left(V_{K^{\sigma}} / K^{\sigma}\right) \oplus \cdots \oplus H_{\mathrm{dR}}^{n}\left(V_{K^{\sigma}}\right) / K^{\sigma^{f-1}}\right)$. Thus, $\bigwedge_{K}\left(H_{\mathrm{dR}} \otimes K\right)_{\underline{a}}$ is isomorphic to

$$
\bigwedge_{K}\left(\left(H_{\mathrm{dR}}\right)_{\underline{a}} \oplus\left(H_{\mathrm{dR}}\right)_{p \underline{a}} \oplus \cdots \oplus\left(H_{\mathrm{dR}}\right)_{p^{f-1} \underline{a}}\right),
$$

where $p^{k} \underline{a}:=\left(p^{k} a_{0}, \ldots, p^{k} a_{n+1}\right)$ and $\left(H_{\mathrm{dR}}\right)_{p^{k} \underline{a}}$ is the $p^{k} \underline{a}$-part of $H_{\mathrm{dR}}$ (not of $H_{\mathrm{dR}} \otimes K$ as before), i.e., the sub- $K$-vector space of $H_{\mathrm{dR}}$ on which $\underline{\zeta} \in S$ acts by 
$\left(p^{k} \underline{a}\right)(\underline{\zeta})$. Note that $\operatorname{dim}_{K}\left(H_{\mathrm{dR}}\right)_{p^{k} \underline{a}}=1$. By [D, Proposition 7.6], we have

$$
\operatorname{Fil}^{i}\left(H_{\mathrm{dR}}\right)_{\underline{a}}= \begin{cases}\left(H_{\mathrm{dR}}\right)_{\underline{a}} & i \leq\langle\underline{a}\rangle-1 \\ 0 & i \geq\langle\underline{a}\rangle .\end{cases}
$$

Therefore, the only jump of the Hodge filtration on $\bigwedge_{K}\left(H_{\mathrm{dR}} \otimes K\right)_{\underline{a}}$ happens at the degree

$$
\begin{aligned}
(\langle\underline{a}\rangle & -1)+\cdots+\left(\left\langle p^{f-1} \underline{a}\right\rangle-1\right) \\
& =\sum_{i=0}^{n+1}\left\{\frac{\left\langle a_{i}\right\rangle}{d}\right\}+\sum_{i=0}^{n+1}\left\{\frac{p\left\langle a_{i}\right\rangle}{d}\right\}+\cdots+\sum_{i=0}^{n+1}\left\{\frac{p^{f-1}\left\langle a_{i}\right\rangle}{d}\right\}-f .
\end{aligned}
$$

On the other hand, elementary calculation [W, Lemma 6.14] says that $\left\{\frac{n}{q-1}\right\}+\left\{\frac{p n}{q-1}\right\}+\cdots+\left\{\frac{p^{f-1} n}{q-1}\right\}=\frac{1+p+\cdots+p^{f-1}}{q-1} s(n)=\frac{1}{p-1} s(n)$. Then, by noting $\frac{p^{k}\left\langle a_{i}\right\rangle}{d}$ $=\frac{((q-1) / d) p^{k}\left\langle a_{i}\right\rangle}{q-1}$ and $d$ divides $q-1$, the above quantity is equal to

$(*)_{\text {Hodge }}$

$$
\frac{1}{p-1} \sum_{i=0}^{n+1} s\left(\frac{(q-1)\left\langle a_{i}\right\rangle}{d}\right)-f .
$$

\section{Crystalline Side (Newton Polygon)}

First, we introduce some notations (See also [D, pp. 84-85]). Let $\mathfrak{p}$ denote the prime ideal in $\mathbf{Q}\left(\mu_{d}\right)$ over $p$ corresponding to the embedding $\mathbf{Q}\left(\mu_{d}\right) \hookrightarrow$ $\mathbf{Q}_{p}\left(\mu_{d}\right)$. The reduction modulo $\mathfrak{p}$ defines an isomorphism $\left(\mathbf{Q}\left(\mu_{d}\right) \supset\right) \mu_{d}\left(\mathbf{Q}\left(\mu_{d}\right)\right) \stackrel{\sim}{\rightarrow}$ $\mu_{d}\left(\mathbf{F}_{q}\right)\left(\subset \mathbf{F}_{q}\right)$. We set $t$ to be its inverse. We fix $\underline{a} \in X(S)$ with $a_{0}, \ldots, a_{n+1} \neq 0$. We define a character $\varepsilon_{i}: \mathbf{F}_{q}^{\times} \rightarrow \mu_{d}$ to be

$$
\varepsilon_{i}(x):=t\left(x^{(1-q) / d}\right)^{a_{i}}
$$

(Note that $x^{(1-q) / d}$ lives in $\mu_{d}\left(\mathbf{F}_{q}\right)$ ). Then $\prod_{i=0}^{n+1} \varepsilon_{i}\left(x_{i}\right)$ is well-defined for $\left(x_{0}: \cdots: x_{n+1}\right) \in \mathbf{P}^{n+1}\left(\mathbf{F}_{q}\right)$, since $\prod_{i=0}^{n+1} \varepsilon_{i}=1$. We define a Jacobi sum

$$
J\left(\varepsilon_{0}, \ldots, \varepsilon_{n+1}\right):=(-1)^{n} \sum_{\left(x_{0}: \cdots: x_{n+1}\right) \in \mathbf{P}^{n+1}\left(\mathbf{F}_{q}\right), x_{0}+\cdots+x_{n+1}=0} \prod_{i=0}^{n+1} \varepsilon_{i}\left(x_{i}\right) \in \mathbf{Q}\left(\mu_{d}\right),
$$

where we put $\varepsilon_{i}(0):=0$. Let $\psi$ be a non-trivial additive character $\psi: \mathbf{F}_{q} \rightarrow \mathbf{Q}\left(\mu_{q}\right)$, and we define Gauss sums

$$
g\left(\mathfrak{p}, a_{i}, \psi\right):=-\sum_{x \in \mathbf{F}_{q}} \varepsilon_{i}(x) \psi(x) \in \mathbf{Q}\left(\mu_{d}, \mu_{q}\right),
$$

and

$$
g(\mathfrak{p}, \underline{a}):=q^{-\langle\underline{a}\rangle} \prod_{i=0}^{n+1} g\left(\mathfrak{p}, a_{i}, \psi\right)
$$


Then [D, Lemma 7.9] says that $J\left(\varepsilon_{0}, \ldots, \varepsilon_{n+1}\right)=q^{\langle\underline{a}\rangle-1} g(\mathfrak{p}, \underline{a})$, hence in particular $g(\mathfrak{p}, \underline{a})$ is independent of $\psi$, and lands in $\mathbf{Q}\left(\mu_{d}\right)$. By [D, Proposition 7.10] and the comparison of the Lefschetz trace formula for crystalline cohomology and étale cohomology, the $K$-linear Frobenius action $\varphi^{f}$ on $\left(H_{\text {crys }} \otimes K\right)_{\underline{a}}$ is given by the multiplication by

$$
q^{\langle\underline{a}\rangle-1} g(\mathfrak{p}, \underline{a})=q^{-1} \prod_{i=0}^{n+1} g\left(\mathfrak{p}, a_{i}, \psi\right)
$$

Then, (an essential part of) Stickelberger's theorem [W, Proposition 6.13] says that

$$
(*)_{\text {Newton }} \quad v_{p}\left(q^{-1} \prod_{i=0}^{n+1} g\left(\mathfrak{p}, a_{i}, \psi\right)\right)=\frac{1}{p-1} \sum_{i=0}^{n+1} s\left(\frac{(q-1)\left\langle a_{i}\right\rangle}{d}\right)-f,
$$

where the valuation $v_{p}$ is normalised as $v_{p}(p)=1$. Then, the quantity $(*)_{\text {Hodge }}$ in Section 2 coincides with the quantity $(*)_{\text {Newton }}$ in Section 3 . This means the weakly admissibility of the filtered $\varphi$-module $\left(\left(H_{\mathrm{dR}} \otimes K\right)_{\underline{a}},\left(H_{\text {crys }} \otimes K\right)_{\underline{a}}\right)$.

\section{Acknowledgment}

The author thanks Noriyuki Otsubo for the discussions on Fermat varieties and Jacobi sums. He also thanks the executives in TOYOTA CRDL, Inc. for offering him a special position in which he can concentrate on pure math research. He sincerely thanks Sakichi Toyoda for his philosophy, and the (ex-)executives (especially Noboru Kikuchi, Yasuo Ohtani, Takashi Saito and Satoshi Yamazaki) for inheriting it from Sakichi Toyoda for 80 years after the death of Sakichi Toyoda. He also thanks Shigefumi Mori for intermediating between TOYOTA CRDL, Inc. and the author, and for negotiating with TOYOTA CRDL, Inc. for him. He also heartily thanks Akio Tamagawa and Shinichi Mochizuki for the constant encouragements.

\section{References}

[BO] P. Berthelot, P.; A. Ogus, F-Isocrystals and De Rham Cohomology I. Invent. Math. 72 (1983), 159-199.

[D] P. Deligne, Hodge cycles on abelian varieties. in Hodge Cycles, Motives, and Shimura Varieties, Lecture Notes in Math. 900, Springer Verlag (1982), 9-100.

[F] J.-M. Fontaine, Représentations $p$-adiques semi-stables. Periodes $p$-adiques (Bures-sur-Yvette, 1988). Astérisque 223 (1994), 113-183. 
[T] T. Tsuji, p-adic étale cohomology and crystalline cohomology in the semistable reduction case. Invent. Math. 137 (1999), 233-411.

[W] L. Washinghton, Introduction to Cyclotomic Fields. Springer-Verlag, New York, 1997.

Research Institute for Mathematical Sciences

Kyoto University

Kyoto, 606-8502, Japan

E-mail: gokun@kurims.kyoto-u.ac.jp 